\title{
Editorial: Anomalous Transport: Applications, Mathematical Perspectives, and Big Data
}

\author{
Carlos Mejía-Monasterio ${ }^{1 *}$, Ralf Metzler ${ }^{2}$ and Jürgen Vollmer ${ }^{3}$ \\ ${ }^{1}$ Laboratory of Physical Properties, School of Agricultural, Food and Biosystems Engineering, Technical University of Madrid, \\ Madrid, Spain, ${ }^{2}$ Institute for Physics and Astronomy, University of Potsdam, Potsdam-Golm, Germany, ${ }^{3}$ Institut für Theoretische \\ Physik, Universität Leipzig, Leipzig, Germany
}

Keywords: anomalous (or non-Fickian) diffusion, anomalous heat conduction, stochastic dynamics, molecular overcrowding, dynamical systems

\section{Editorial on the Research Topic}

Anomalous Transport: Applications, Mathematical Perspectives, and Big Data

Diffusion is a fundamental process that addresses the stochastic motion of a test particle and the emerging broadening of its distribution function in the course of time. Diffusive-like motion was first reported in 1828 following the seminal experiments by Robert Brown a year earlier on the erratic motion of micron-sized granules contained in Clarkia pulchella pollen grains [1]. The theoretical foundations of Brown's observations were established almost a century later by Einstein, Sutherland, Smoluchowski, and Langevin [2-5].

Diffusion theory is a fundamental and well established area of research which continues to be very

\section{OPEN ACCESS}

Edited and reviewed by: Alex Hansen, Norwegian University of Science and Technology, Norway

*Correspondence: Carlos Mejia-Monasterio carlos.mejia@upm.es

Specialty section:

This article was submitted to Interdisciplinary Physics,

a section of the journal

Frontiers in Physics

Received: 28 October 2020 Accepted: 29 October 2020 Published: 23 November 2020

Citation:

Mejia-Monasterio C, Metzler $R$ and Vollmer J (2020) Editorial: Anomalous Transport: Applications, Mathematical

Perspectives, and Big Data. Front. Phys. 8:622417. doi: 10.3389/fphy.2020.622417 active. Concurrently, a broad range of stochastic phenomena exhibit significant deviations from normal diffusive behavior, commonly termed as anomalous diffusion. Anomalous transport is ubiquitously observed in many complex systems, ranging from the electronic transport of solid-state disordered systems [6], the motion of molecules inside living cell [7] and on their membrane [8], in the telomere motion inside the nucleus of mammalian cells [9], in soil transport [10], in heat transport in low-dimensional systems [11], and in certain classes of billiards [12], among a host of other phenomena.

From a statistical view point, all these diverse phenomena share a common description depending on how the broadening of the distribution function of the process $\xi(t)$ grows in time. This is customarily measured by the variance of the second moment, the so-called mean squared displacement $(\mathrm{MSD})\left\langle\xi^{2}(t)\right\rangle \simeq t^{\gamma}$. Normal diffusion corresponds to an MSD that grows linearly in time $\gamma=1$, while anomalous diffusion is classified as subdiffusive for $\gamma<1$ and superdiffusive $\gamma>1$. From a dynamical view, there is a wealth of theoretical research devoted to unveil the physical mechanisms that can induce anomalous diffusion (see, e.g., Refs. 13-15 and references therein).

From an experimental point of view the past decade has vividly changed the field. Video microscopy and particle tracking are providing a rapidly increasing wealth of highly-resolved experimental observations. Moreover, numerical simulations of ensembles of trajectories are now feasible also for disordered systems where one must average over many realisations of the geometry. The resulting data sets should best be addressed from a big data perspective to extract characteristic transport properties. Firstly, this poses challenges for the automatic data processing and parameter inference. Secondly, it calls for new mathematical perspectives that underpin the data analysis from a unified point of view. In particular, data sets are big enough now to address the anomalous decay of correlations in the dynamics and to search for universality in the transport. 
The aim of this Research Topic attempts to unify different visions, approaches and methodologies around the field of anomalous diffusion, to present an overview of the state of the art of the field, and insight into the main directions of current research. The research topic comprises 19 contributions discussing current issues, from experiments to theory, in anomalous transport. Here is a brief overview of these contributions.

A pathway to obtain anomalous diffusion is through molecular crowding and confinement. These two constraints prevail in living cells, where anomalous transport is often observed. With the advent of experimental techniques such as Fluorescence Spectroscopy (FS) and Single-Particle Tracking (SPT) we are able to study the dynamics in the cell and the cell kinetics that determine the essential processes of the living matter. This is the subject of the perspective article by Wringer et al. After an extensive overview Wringer et al. propose a collaborative global challenge to unify data benchmarks of biologically-relevant measures.

Memory effects have in general a strong influence on transport, leading to non-stationarity and aging. Using spectral theory Lapolla et al. discuss the non-Markovian stochastic processes that arises in the projected dynamics, like, e.g., in coarse-graining. Following this approach and using the coordinate Bethe ansatz Lapolla et al. are able to solve the problem of single file diffusion in a tilted box, where projection corresponds to the dynamics of the tagged particle after tracing out the dynamics of the rest of the particles.

Masó-Puigdellosas et al. also consider memory effects of resetting dynamics. They study how anomalous diffusion exhibited by otherwise diffusive random walks is determined when the walkers are subject to random relocations with memory. They show that the type of transport, from subdiffusive to superdiffusive is determined by the details of the resetting dynamics.

Over the last few decades different classes of anomalous transport have been described and observed. They include situations in which the MSD grows linearly in time as in normal diffusion, yet the statistics of the displacement is described by a strongly non-Gaussian probability distribution function. Such a situation, known as Brownian yet non-Gaussian diffusion (BnGD), is often observed in soft matter, biological and complex systems, and in general, in transport through heterogeneous environments [16]. Generalisations to nonGaussian fluctuations include diffusing diffusivities [17], and strong anomalous diffusion in which the time scaling of the moments of the displacement is nonlinear in the moment's order [18].

Brownian yet non-Gaussian diffusion is the subject of the article of Baldovin et al. in which the authors consider a polymerization process in a closed fixed volume and with a fixed number of polymers. Following the statistics of the polymers center of mass they show that BnGD is a direct consequence of the polymerization process.

Di Tullio et al. discuss how anomalous diffusion emerges from diffusive dynamics on complex heterogeneous environments. They consider different models of anomalous diffusion such as continuous time random walks, Brownian motion with grey noise, and time-subordinated processes with a heterogeneous condition in which spatial and temporal scales are in turn random variables following a proper distribution, and show how this rescaling determines the type of anomalous transport.

Heterogeneous diffusion is characterised by an initial sampling of the local environment to a later exploration of the surrounding effective medium, yielding generically transient anomalous diffusion. Spakowitz studies the emergence of anomalous diffusion in heterogeneous environments. $\mathrm{He}$ models heterogeneity with a diffusivity that varies with position. By means of diagrammatic techniques, Spakowitz obtains an exact expression for the particle Green's function that captures the spatially varying diffusivity.

Active microrheology studies the response of a complex medium to the passage of a tracer particle that is driven through it, as in a colloid driven by an external force through a quiescent bath. The tracer particle reaches a stationary state in which the external force and the dragging force equilibrate. In the stationary state the tracer particle produces a nonequilibrium inhomogeneity in the density of the medium which in general leads to anomalous diffusion of the tracer [19].

Werner et al. consider the transport of asymmetrically grafted nanoparticles, often called hairy nanoparticles, that are pulled through a quiescent bath. Because of the external pulling the grafted colloids change the depletion zone around them, which in turns leads to an anisotropic effective viscosity. Werner et al. study the dependence of the friction force exerted by the bath on the profile of the local viscosity, and discuss the consequences of their results for active microrheology.

Bacteria are an example of active matter in which each bacterium transduces chemical energy into motion. Therefore, active matter is intrinsically out of equilibrium. What happens when a bacteria cell moves across complex geometries? This is the topic that Weber et al. address in their article. It is known that bacteria strongly interact with their surroundings and are often guided by confinements. Weber et al. consider a bacteria moving inside a labyrinth with square lattice geometry. The bacteria's motion is modelled as a run-and-tumble. Their analytical predictions show a transient non-Gaussian diffusion and compare well with experiments.

In the article of Um et al. active matter is further investigated. Modelling self-propelled particles as a Langevin dynamic driven by a telegraphic active noise, which essentially describes the transport of an active particle under confinement. Um et al. show that depending on the properties of the noise, different types of transport emerge, and they discuss relations with runand-tumble and Lévy walks.

Reza Shaebani et al. consider the run-and-tumble model, characteristic of bacteria's motion and derive an expression for the MSD. They show that depending on the dynamic parameters and on the initial position of the process, the MSD exhibits a variety of transient regimes of anomalous transport. In all cases diffusion sets in asymptotically, with a diffusion coefficient that turns out to be independent of the initial starting of the process.

Territoriality is ubiquitous in the animal kingdom. Among mammals it is often associated with individuals marking their 
passage, to which other individuals react upon their encounter. A simple model for this situation is the so-called Territorial Random Walk (TRW) which comprises diffusion and local avoidance mechanism. Sarvaharman et al. consider a correlated TRW in which walkers are persistent and analyse the phase diagram picture that arises in this situation.

Transport in biological systems occurs in a complex "disordered" environment characterised by geometric disorder and the existence of energy barriers. For instance, inside the cell the actin cytoskeleton forms a cellular compartmentalisation for the proteins diffusing in the plasma membrane. It is believed that such constraints are responsible for the commonly observed subdiffusion. Muñoz-Gil et al. discuss the dynamics of a random walk in a compartmentalisation substrate where compartments are separated by barriers with random transmittance. They show that in their model a stochastic transmittance is essential to anomalous diffusion.

Segovia Gutierrez et al. present experimental results for the dynamics of synthetic trimer molecules in a random potential energy landscape that is realised by a random light field. Using SPT techniques they study the translational and rotational MSD showing that trimers move subdiffusively. However, such anomalous behavior is transient and converges to normal diffusion in the long time limit.

While anomalous diffusion has been well established over decades, anomalous heat transport was experimentally observed for the first time in 2008 [20]. Almost 200 years ago Joseph Fourier established from simple physical assumptions that the transport of heat must be described by a diffusion equation. Even when this is overwhelmingly observed, a first principles derivation of Fourier's law of heat conduction is still absent.

In the attempts to derive the diffusive character of heat conduction it has turned out that in low-dimensional systems Fourier's law probably does not hold. From a theoretical viewpoint, anomalous heat transport arises in general due to the strong dynamic correlations existing in low-dimensional systems, though a detailed understanding has remained elusive for decades. An overview of these issues appear in the review article of Benenti et al. They discuss anomalous heat transport in anharmonic chains of oscillators, considering magnetic fields and long-range interactions, among others. Furthermore, they discuss the important case of coupled transport which is the essence of generic thermodynamic transport. Benenti et al. close their review with an overview of open problems and future perspectives.

Concerning one-dimensional systems, the current picture that emerges is that when heat transport turns anomalous it means that the heat conductivity depends on the global state of the system rather than on its local properties. In the review article of Dhar et al. the authors discuss this non-locality, due to which Fourier's law is replaced by a non-local fractional diffusion equation. Dhar et al. review the different theoretical

\section{REFERENCES}

1. Brown R. A brief account of microscopical observations made in the months of June, July and August 1827, on the particles contained in the pollen of plants; approaches leading to this framework and offer an exhaustive overview of recent progress.

Giberti et al. further discuss the non-locality implied by an anomalous heat conduction and how this affects the important assumption of Local Thermal Equilibrium (LTE). The non-local description of heat transport has direct fundamental consequences on the definition of local intensive thermodynamic quantities and therefore, on the LTE. To study these consequences Giberti et al. show that onedimensional chains of oscillators interacting through a Lennard-Jones potential exhibit anomalously large distortions and fluctuations that hinder an appropriate thermodynamic treatment.

By means of parameter estimation from a finite set of trajectories and machine learning techniques Ridha Znaidi et al. provide algorithmic strategies to uncover the underlying dynamics of such trajectories. They assume the dynamics to be described by a set of fractional partial differential equations, which in the extended systems are able to describe anomalous diffusion.

Finally, the automatic data processing and parameter inference is discussed by Natole et al. For any scoring function, the area under the receiver operating characteristic curve (AUC) is equivalent to the probability of a positive sample ranking higher than a negative sample. As such the AUC is a standard classification measure used in the analysis of imbalance class data and in clinical trials such as the development of vaccines to mitigate the COVID-19 pandemic. In their article Natole et al. develop a stochastic learning algorithm that maximises the AUC with respect to accuracy.

We are confident that this collection of articles provides a timely showcase for the state of the art in the field of anomalous diffusion. Moreover we look forward to see how the topics presented here will inspire further work in this rapidly developing field.

\section{AUTHOR CONTRIBUTIONS}

All authors listed have made a substantial, direct, and intellectual contribution to the work and approved it for publication.

\section{FUNDING}

CM-M acknowledges financial support from the Spanish Government grant PGC2018-099944-B-I00 (MCIU/AEI/ FEDER, UE). RM acknowledges the Foundation for Polish Science (Fundacja na rzecz Nauki Polskiej, FNP) for a Humboldt Honorary Polish Research Scholarship.

and on the general existence of active molecules in organic and inorganic bodies. Phil Mag (1828) 4:161-73. doi:10.1080/14786442808674769.

2. Einstein AA. Über die von der molekularkinetischen Theorie der Wärme geforderte Bewegung von in ruhenden Flüssigkeiten suspendierten TeilchenÜber die von der molekularkinetischen Theorie der Wärme 
geforderte Bewegung von in ruhenden Flüssigkeiten suspendierten Teilchen. Ann Phys (1905) 322:549-60. doi:10.1002/andp.19053220806.

3. Sutherland W. LXXV. a dynamical theory of diffusion for nonelectrolytes and the molecular mass of albumin. Lond Edinb Dubl Phil Mag (1905) 9:781-5. doi:10.1080/14786440509463331.

4. von Smoluchowski M. Zur kinetischen Theorie der Brownschen Molekularbewegung und der Suspensionen. Ann Phys (1906) 326:756-80. doi:10.1002/andp.19063261405.

5. Langevin P. Sur la théorie de movement brownien. Compt. Rend. Acad. Sci (1908) 146:530-3.

6. Bouchaud JP, Georges A. Anomalous diffusion in disordered media: statistical mechanisms, models and physical applications. Phys Rep (1990) 195:127-293. doi:10.1016/0370-1573(90)90099-n.

7. Saxton MJ. Anomalous subdiffusion in fluorescence photobleaching recovery: a Monte Carlo study. Biophys J (2001) 81:2226-40. doi:10.1016/s0006-3495(01) 75870-5.

8. Nicolau DV, Hancock JF, Burrage K. Sources of anomalous diffusion on cell membranes: a Monte Carlo study. Biophys J (2007) 92:1975-87. doi:10.1529/ biophysj.105.076869.

9. Bronstein I, Israel Y, Kepten E, Mai S, Shav-Tal Y, Barkai E, et al.. Transient anomalous diffusion of telomeres in the nucleus of mammalian cells. Phys Rev Lett (2009) 103:018102. doi:10.1103/physrevlett.103.018102.

10. Martin RL, Jerolmack DJ, Schumer R. The physical basis for anomalous diffusion in bed load transport. J Geophys Res Earth Surface (2012) 117: 01018. doi:10.1029/2012jf002608.

11. Lepri S, Livi R, Politi A. Thermal conduction in classical low-dimensional lattices. Phys Rep (2003) 377:1-80. doi:10.1016/s0370-1573(02)00558-6.

12. Armstead DN, Hunt BR, Ott E. Anomalous diffusion in infinite horizon billiards. Phys Rev E (2003) 67:021110. doi:10.1103/physreve.67.021110.

13. Metzler R, Klafter J. The random walk's guide to anomalous diffusion: a fractional dynamics approach. Phys Rep (2000) 339:1-77. doi:10.1016/s0370-1573(00)00070-3.
14. Klages R, Radons G, Sokolov IM. Anomalous transport: foundations and applications. Weinheim, Germany: Wiley-VCH (2008)

15. Sokolov IM. Models of anomalous diffusion in crowded environments. Soft Matter (2012) 8:9043-52. doi:10.1039/c2sm25701g.

16. Wang B, Kuo J, Bae SC, Granick S. When Brownian diffusion is not Gaussian. Nat Mater (2012) 11:481-5. doi:10.1038/nmat3308.

17. Chechkin AV, Seno F, Metzler R, Sokolov IM. Brownian yet non-Gaussian diffusion: from superstatistics to subordination of diffusing diffusivities. Phys Rev X (2017) 7:021002. doi:10.1103/PhysRevX.7.021002.

18. Castiglione P, Mazzino A, Muratore-Ginanneschi P, Vulpiani A. On strong anomalous diffusion. Phys Nonlinear Phenom (1999) 134:75-93. doi:10.1016/ s0167-2789(99)00031-7.

19. Bénichou O, Illien P, Mejía-Monasterio C, Oshanin G. A biased intruder in a dense quiescent medium: looking beyond the force-velocity relation. J. Stat. Mech (2013) 2013:P05008. doi:10.1088/1742-5468/2013/05/ p05008.

20. Chang CW, Okawa D, Garcia H, Majumdar A, Zettl A. Breakdown of Fourier's law in nanotube thermal conductors. Phys Rev Lett 101 (2008) 075903. doi:10. 1103/PhysRevLett.101.075903.

Conflict of Interest: The authors declare that the research was conducted in the absence of any commercial or financial relationships that could be construed as a potential conflict of interest.

Copyright (0) 2020 Mejía-Monasterio, Metzler and Vollmer. This is an open-access article distributed under the terms of the Creative Commons Attribution License (CC $B Y$ ). The use, distribution or reproduction in other forums is permitted, provided the original author(s) and the copyright owner(s) are credited and that the original publication in this journal is cited, in accordance with accepted academic practice. No use, distribution or reproduction is permitted which does not comply with these terms. 